\title{
In vitro and in vivo antischistosomal activity of ferroquine derivatives
}

\author{
Jennifer Keiser ${ }^{1,2^{*}}$, Mireille Vargas ${ }^{1,2}$, Riccardo Rubbiani ${ }^{3}$, Gilles Gasser ${ }^{3}$ and Christophe Biot ${ }^{4,5}$
}

\begin{abstract}
Background: Schistosomiasis is a neglected tropical disease and drug-repurposing is a useful strategy to fill its exhausted drug development pipeline. The ferrocenyl analogue of chloroquine, ferroquine, is an antimalarial in late-stage drug development. The aim of the present work was to study the antischistosomal activity of ferroquine against Schistosoma mansoni adult worms and newly transformed schistosomula (NTS) in vitro and in vivo. Hydroxyl-ferroquine and ruthenoquine were included to study the potential role of reactive oxygen species in the antischistosomal activity. Chloroquine and mefloquine, the later described for its antischistosomal properties, served as comparators.

Findings: All metal complexes were shown to be moderately cytotoxic on human cervix HeLa cancer cells and human fetal lung fibroblasts MRC-5. 72 hours post-incubation NTS exposed to $33.3 \mu \mathrm{M}$ ruthenoquine had died, while ferroquine and hydroxyl-ferroquine treated worms were strongly affected. No activity was observed treating NTS with chloroquine at $33.3 \mu \mathrm{M}$. Incubation of adult S. mansoni with $33.3 \mu \mathrm{M}$ of the organometallic derivatives were highly affected in viability but were still alive 72 hours post-incubation. Mefloquine showed the highest activity against NTS and adult S. mansoni. Low total worm burden reductions of 0-36\% were observed following oral administration of $200-800 \mathrm{mg} / \mathrm{kg}$ of the ferroquine derivatives to S. mansoni-infected mice.

Conclusions: The organometallic compounds evaluated in this study revealed moderate in vitro activity against both larval and adult stages of S. mansoni but low in vivo activity. No correlation can be drawn between the antimalarial and antischistosomal activity of chloroquine analogues and oxidative shock does not seem to play a role in the activity of these compounds against $S$. mansoni.
\end{abstract}

Keywords: Schistosoma mansoni, Ferroquine, Chloroquine, Ruthenoquine, Hydroxyl-ferroquine, In vitro, In vivo

\section{Background}

Schistosomiasis is a neglected tropical disease, which causes a considerable public health burden [1]. Millions of people require treatment each year to prevent the considerable health effects of schistosomiasis. For example, in 2012 it has been estimated that 249 million people should be treated in preventive chemotherapy programs [2]. Yet, treatment and control of schistosomiasis relies on a single drug, namely praziquantel $[1,3,4]$.

Research on novel antischistosomal drugs is nearly exclusively driven by academic groups with a strong focus on phenotypic approaches, since protein targets

\footnotetext{
* Correspondence: jennifer.keiser@unibas.ch

'Department of Medical Parasitology and Infection Biology, Swiss Tropical and Public Health Institute, Basel, Switzerland

¿University of Basel, Basel, Switzerland

Full list of author information is available at the end of the article
}

are almost unknown [5]. Over the past years, libraries consisting of existing drugs, so called drug-repurposing, were investigated in order to fill the exhausted drug development pipeline for schistosomiasis [6]. Drug repurposing circumvents the high cost of drug discovery and development, the high failure rates and the long duration to develop novel treatments by finding new uses for compounds other than those they were initially intended to treat [7]. Antimalarials, in particular, have been well studied, which is not surprising since both Plasmodium and schistosomes degrade hemoglobin [4]. For example, the excellent antischistosomal activity of mefloquine (MQ, Figure 1) was demonstrated in S. haematobium, S. japonicum and S. mansoni rodent models [8]. The promising results obtained with MQ in laboratory studies were followed up in clinical trials in the past years $[9,10]$. Over the last decade, there has 


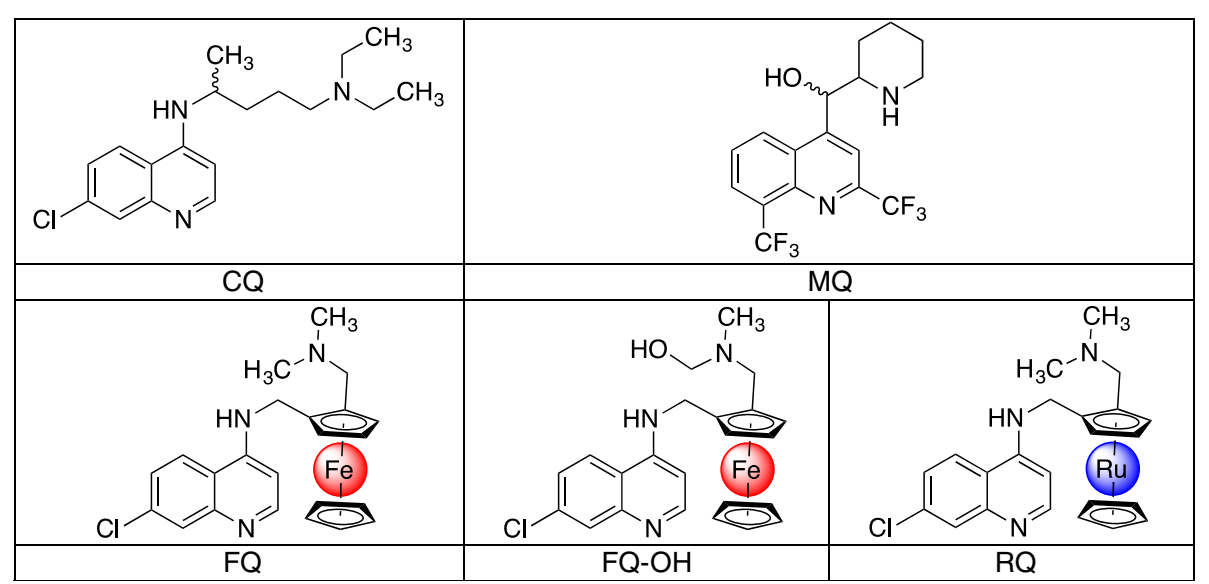

Figure 1 Chemical structures of chloroquine (CQ), mefloquine (MQ), ferroquine (FQ), hydroxyl-ferroquine (FQ-OH) and ruthenoquine (RQ).

been increasing interest in metal-based drugs and numerous metal complexes have been synthesized and evaluated as antimalarial agents [11]. The most promising candidate was undoubtedly the ferrocenyl analogue of chloroquine ( $\mathrm{CQ}$, Figure 1), ferroquine (FQ, Figure 1). FQ reached two different Phase IIb clinical trials, in combination with artesunate or OZ439, for the treatment of uncomplicated Plasmodium falciparum malaria (http://www.mmv.org/research-development/projectportfolio/oz439fq). FQ has a specific parasiticidal effect on Plasmodium due to the FQ-induced oxidative stress within the digestive vacuole and the subsequent destruction of the membrane resulting in death of the parasite [12].

The aim of the present work was to study the antischistosomal activities of FQ against $S$. mansoni in vitro and in vivo. In addition, we investigated whether redox activation plays a role in antischistosomal activity by including the ruthenocenyl analogue of $\mathrm{FQ}$, namely ruthenoquine ( $R Q$, Figure 1), which was shown, contrary to $\mathrm{FQ}$, to be unable to produce reactive oxygen species (ROS) in P. falciparum [13]. This difference in redox activity was assumed to be responsible for the greater antimalarial activity of FQ over RQ. Furthermore, the antischistosomal activity of hydroxyl-ferroquine (FQ-OH, Figure 1) was also studied since FQ-OH is also able to produce hydroxyl radicals and provides reduced cytotoxic effects compared to FQ [14].

\section{Findings}

\section{Methods}

\section{Animals and parasites}

In vivo studies were carried out in accordance with Swiss national and cantonal regulations on animal welfare (permission no. 2070) at the Swiss Tropical and Public Health Institute (Basel, Switzerland). Female mice (NMRI strain, $\mathrm{n}=31$; weight $\sim 20-22 \mathrm{~g}$ ) were purchased from Charles River, Germany, kept under environmentallycontrolled conditions (temperature $\sim 25^{\circ} \mathrm{C}$; humidity $\sim 70 \%$; 12-hour light and 12-hour dark cycle) with free access to water and rodent diet and acclimatized for one week before infection. Cercariae of S. mansoni were obtained from infected intermediate host snails (Biomphalaria glabrata) as described previously [15].

\section{Compounds}

FQ, FQ-OH and RQ were synthesized according to reported procedures $[13,14,16]$. MQ was kindly obtained from Mepha AG (Aesch, Switzerland). CQ was purchased from Sigma (Buchs, Switzerland). For in vitro studies, compounds were dissolved in DMSO (Fluka, Buchs, Switzerland) to obtain stock solutions of $10 \mathrm{mg} / \mathrm{ml}$. For in vivo studies, compounds were suspended in $7 \%(\mathrm{v} / \mathrm{v})$ Tween 80 and $3 \%(\mathrm{v} / \mathrm{v})$ ethanol shortly before oral treatment $(10 \mathrm{ml} / \mathrm{kg})$ of mice.

\section{In vitro studies}

\section{Newly transformed schistosomula (NTS)}

S. mansoni cercariae were mechanically transformed to newly transformed schistosomula (NTS) [17]. A NTS suspension at a concentration of 100 NTS per $50 \mu \mathrm{l}$ was prepared using Medium 199 (Invitrogen, Carlsbad, CA) [supplemented with $5 \%$ inactivated fetal calf serum (iFCS) and $100 \mathrm{U} / \mathrm{ml}$ penicillin and $100 \mathrm{mg} / \mathrm{mL}$ streptomycin (Invitrogen). NTS were incubated with $10 \mu \mathrm{M}$ and $33.3 \mu \mathrm{M}$ of the test compounds for $72 \mathrm{~h}$. Compounds were tested at least in triplicate and the highest concentration of DMSO served as control. Plates were incubated at $37^{\circ} \mathrm{C}, 5 \% \mathrm{CO}_{2}$. NTS were evaluated by microscopic readout (Carl Zeiss, Germany, magnification 80x) using a viability scale scoring death, changes in motility, viability, and morphological alterations [17]. 


\section{Adult S. mansoni}

Adult schistosomes obtained from infected mice were incubated in the presence of $10 \mu \mathrm{M}$ and $33.3 \mu \mathrm{M}$ of the test compounds for up to $72 \mathrm{~h}$. Phenotypes were monitored daily based on motility, viability and morphological alterations under an inverse microscope (Carl Zeiss, Germany, magnification $80 \times$ ).

\section{Cytotoxicity studies}

Cytotoxicity studies were performed on human cervix HeLa cancer cells and non tumorigenic human fetal lung fibroblasts MRC-5 to compare the activity of FQ, RQ, FQ-OH, CQ, MQ and cisplatin. The cell viability was determined via a colorimetric cell-based assay using Resazurin (Promocell GmbH). Briefly, one day before treatment cells were plated in triplicates in 96-well plates at a density of $4 \times 10^{3}$ cells/well in $100 \mu$ l. Upon treating cells with increasing concentrations of the target complexes (freshly prepared stock solution in DMSO), cells were incubated at $37^{\circ} \mathrm{C} / 6 \% \mathrm{CO}_{2}$ for $48 \mathrm{~h}$, the medium was removed, and $100 \mu \mathrm{l}$ of complete medium containing resazurin $(0.2 \mathrm{mg} / \mathrm{ml}$ final concentration) was added. After $4 \mathrm{~h}$ of incubation at $37^{\circ} \mathrm{C} / 6 \% \mathrm{CO}_{2}$, the fluorescence of the highly red fluorescent resorufin product was quantified at $590 \mathrm{~nm}$ emission with $540 \mathrm{~nm}$ excitation wavelength in a SpectraMax M5 microplate Reader.

\section{S. mansoni in vivo studies}

Groups of 3-4 NMRI mice were treated orally with single oral doses of $200 \mathrm{mg} / \mathrm{kg}$ of FQ, FQ-OH and RQ. In addition, one group of mice was treated with a single oral dose of $800 \mathrm{mg} / \mathrm{kg}$ FQ. Untreated mice served as controls in all experiments. At $21 \mathrm{~d}$ post-treatment, animals were killed by the $\mathrm{CO}_{2}$ method and dissected. Worms were removed by picking, then sexed and counted as previously described [18].

\section{Statistics}

Parasite viability values of treated and untreated worms obtained from microscopic evaluation were averaged (means (+/- standard deviation)) using Microsoft Excel software. The Kruskal-Wallis test was applied for in vivo studies, comparing the medians of the worm counts of the treatment and control groups. A difference in median was considered to be significant at a significance level of 5\% (StatsDirect statistical software, version 2.7.2.; StatsDirect Ltd., United Kingdom).

\section{Results and discussion Cytotoxicity studies}

We assessed the potential toxicity of the compounds studied in this work on cervical cancer cells (HeLa) and non-cancerous cells (MRC-5). Cisplatin, the best known metal-based drug on the market was used as a reference.
As shown in Table 1, all metal complexes were shown to be moderately cytotoxic. MQ was the most toxic compound in these assays. This is not surprising, since MQ is well known for its adverse events, including gastrointestinal effects [9] and neuropsychiatric toxicity [19]. On the other hand, FQ was well tolerated and shown to be lacking relevant adverse effects on central nervous system, respiratory, renal, and gastrointestinal functions in a phase I trial [20]. However, larger trials are necessary to confirm this finding.

\section{Activity against NTS and adult S. mansoni in vitro}

24 hours post-incubation all NTS exposed to $33.3 \mu \mathrm{M}$ FQ, FQ-OH and RQ showed strongly reduced viabilities. 72 hours post-incubation all NTS exposed to $33.3 \mu \mathrm{M}$ RQ had died, while FQ and FQ-OH treated worms were strongly affected but still alive. Therefore, unlike for P. falciparum and Trypanosoma brucei gambiense [21], oxidative shock does not seem to play a role in the activity of these compounds against S. mansoni, since $R Q$, which cannot produce ROS is the most active of the three organometallic drug against NTS in vitro. This absence of redox activation could be explained by the difference in the target of FQ, FQ-OH and RQ in $S$. mansoni compared to $P$. falciparum. In $P$. falciparum, sour conditions present in the acidic compartment where FQ localizes as well as of $\mathrm{H}_{2} \mathrm{O}_{2}$ are necessary for the formation of $\mathrm{HO}^{*}$ [12]. For comparison, no activity was observed treating NTS with CQ at $33.3 \mu \mathrm{M}$ (Figure 2a) while at this concentration 24 hours post-incubation with MQ all worms had died. The antischistosomal mechanism of action of MQ and CQ is also not known. Note that these 2 drugs do not produce hydroxyl radicals [22]. The antimalarial activity of CQ and MQ is largely attributed to an inhibition of hemoglobin degradation. However, it was recently shown that hemozoin inhibition of $C Q, M Q$ or quinine does not exhibit a correlation with their antischistosomal properties [23]. Furthermore, it was demonstrated that MQ interferes with glycolysis in NTS [24].

\begin{tabular}{|c|c|c|}
\hline Complex & $\mathrm{IC}_{50} \mathrm{MRC}-5(\mu \mathrm{M})$ & $\mathrm{IC}_{50} \mathrm{HeLa}(\mu \mathrm{M})$ \\
\hline Cisplatin & $7.9^{ \pm 1.2}$ & $11.5^{ \pm 2.9}$ \\
\hline $\mathrm{FQ}$ & $24.4^{ \pm 0.9}$ & $10.1^{ \pm 0.3}$ \\
\hline $\mathrm{FQ}-\mathrm{OH}$ & $22.6^{ \pm 1.2}$ & $16.8^{ \pm 1.5}$ \\
\hline $\mathrm{RQ}$ & $21.9^{ \pm 2.6}$ & $8.8^{ \pm 0.41}$ \\
\hline $\mathrm{CQ}$ & $55.1 \pm 1.3$ & $87.0^{ \pm 1.5}$ \\
\hline MQ & $16.7^{ \pm 0.2}$ & $6.7^{ \pm 0.7}$ \\
\hline
\end{tabular}

Cisplatin was used as positive control. Standard deviations are shown in superscript. 

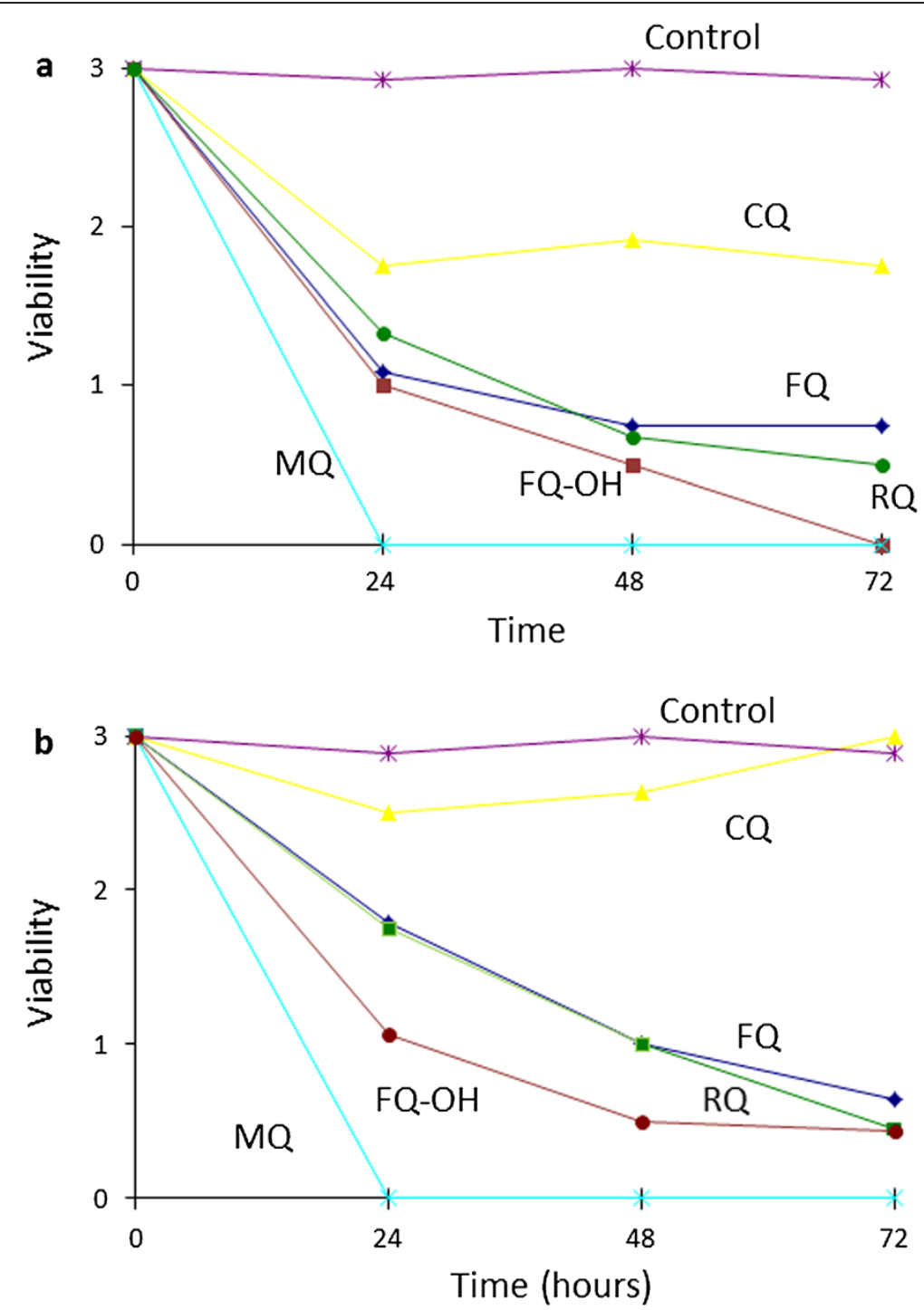

Figure 2 Activity of ferroquine (FQ), hydroxy-ferroquine (FQ-OH), ruthenoquine (RQ), chloroquine (CQ) and mefloquine (MQ) versus untreated control worms in vitro at $33.3 \mu \mathrm{g} / \mathrm{ml}$ against NTS (a) and adult S. mansoni (b).

Exposure to $10 \mu \mathrm{M}$ of the test drugs resulted in reduced motilities of NTS but the larvae did not die (data not shown).

A similar trend was observed on adult S. mansoni. Incubation of adult $S$. mansoni with $33.3 \mu \mathrm{M}$ of MQ resulted in death of worms $24 \mathrm{~h}$ post-treatment, while adult $S$. mansoni exposed to the organometallic derivatives were highly affected in viability but were still alive 72 hours postincubation (Figure 2b). At $10 \mu \mathrm{M}$ adults showed strongly reduced viabilities 48-72 hours post-incubation with all derivatives (data not shown).

\section{Activity against adult S. mansoni in vivo}

Compounds progressed into in vivo studies despite the fact that in vitro activities were only observed at concentrations that were close to cytotoxic concentrations.
However, in our recent studies MQ was well tolerated by mice [8].

We have previously shown that CQ lacked antischistosomal activity in vivo (worm burden reduction of 11\%) [8]. FQ is known for important and additional properties (e.g. its higher lipophilicity) and mechanism of actions (the above mentioned radical formation as well as hemozoin inhibition) compared to CQ [22]. Yet, treatment of mice with 200 and $800 \mathrm{mg} / \mathrm{kg} \mathrm{FQ}$, showed low total worm burden reductions of $19.4 \%$ and $35.6 \%$ (Table 2). One of the mice treated with $800 \mathrm{mg} / \mathrm{kg}$ FQ died within 24 hours post-treatment. No activity was observed treating mice with RQ at $200 \mathrm{mg} / \mathrm{kg}$. Finally, a total worm burden reduction of $17.3 \%$ was observed following treatment with FQ-OH. Hence, modification of CQ by a ferrocenyl or ruthenocenyl fragment does not increase the 
Table 2 Effect on worm burden of single oral doses of three selected organometallic CQ derivatives administered to mice harboring a 49-day-old adult S. mansoni infection, stratified by sex and worm distribution

\begin{tabular}{|c|c|c|c|c|c|c|c|c|c|c|c|c|}
\hline \multirow[t]{2}{*}{ Drug } & \multirow[t]{2}{*}{ Dose $(\mathrm{mg} / \mathrm{kg})$} & \multirow{2}{*}{$\begin{array}{l}\text { No. of mice } \\
\text { investigated }\end{array}$} & \multirow{2}{*}{$\begin{array}{c}\text { No. of } \\
\text { mice cured }\end{array}$} & \multicolumn{5}{|c|}{ Mean number of worms (SD) } & \multirow{2}{*}{$\begin{array}{l}\text { Total worm burden } \\
\text { reduction (\%) }\end{array}$} & \multirow[t]{2}{*}{$p$-value } & \multirow{2}{*}{$\begin{array}{l}\text { Female worm burden } \\
\text { reduction (\%) }\end{array}$} & \multirow[t]{2}{*}{$p$-value } \\
\hline & & & & Liver & $\begin{array}{l}\text { Mesenteric } \\
\text { veins }\end{array}$ & Total & Males & Females & & & & \\
\hline Control $^{1}$ & - & 8 & - & $0.4(0.7)$ & $33.8(10.2)$ & $34.1(10.3)$ & $19.9(7.7)$ & $14.3(4.2)$ & - & - & - & - \\
\hline Control $^{2}$ & - & 8 & - & $0.6(1.2)$ & $25.8(16.7)$ & $26.4(16.7)$ & $14.5(9.3)$ & $11.9(7.7)$ & - & - & - & - \\
\hline $\mathrm{FQ}^{\mathrm{a}}$ & 200 & 4 & 0 & $1.8(2.4)$ & $25.8(7.3)$ & $27.5(7.3)$ & $16.3(4.9)$ & $11.3(4.0)$ & 19.4 & $>0.05$ & 21.0 & $>0.05$ \\
\hline $\mathrm{FQ}^{\mathrm{b}}$ & 800 & $4^{*}$ & 0 & $0.7(1.2)$ & $16.3(4.2)$ & $17.0(5.3)$ & $10.3(5.8)$ & $6.7(1.2)$ & 35.6 & $>0.05$ & 43.7 & 0.018 \\
\hline $\mathrm{FQ}-\mathrm{OH}^{\mathrm{a}, \mathrm{b}}$ & 200 & 3 & 0 & $3.7(5.5)$ & $21.3(26.6)$ & $25.0(24.4)$ & $16.3(14.3)$ & $8.7(10.8)$ & 17.3 & $>0.05$ & 33.6 & $>0.05$ \\
\hline $\mathrm{RQ}^{\mathrm{a}}$ & 200 & 4 & 0 & $0.25(0.5)$ & $36.5(10.7)$ & $36.8(10.4)$ & $18.8(5.3)$ & $18.0(5.3)$ & 0 & $>0.05$ & 0 & $>0.05$ \\
\hline MQ & 200 & 5 & 0 & & & & & & $72.3[8]$ & & 100 & \\
\hline CQ & 200 & 5 & 0 & & & & & & $11.7[8]$ & & 93.0 & \\
\hline
\end{tabular}

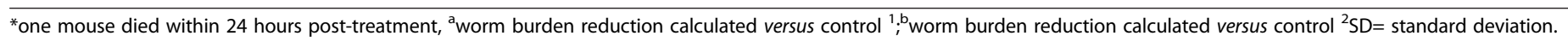


antischistosomal properties of CQ. For comparison, at $200 \mathrm{mg} / \mathrm{kg}$ MQ achieved a much higher worm burden reduction of $72.3 \%$ in $S$. mansoni-infected mice [8]. A few issues of our in vivo results are worth highlighting: interestingly, a moderate female worm burden reduction of $43.7 \%$ ( $\mathrm{p}=0.018$ ) was observed using FQ at $800 \mathrm{mg} / \mathrm{kg}$ (which had not been observed with CQ). A higher effect against female adult S. mansoni was also observed in MQ treated mice [8] pointing to a sex-specific interference of these drugs with the target. Furthermore, in one of the FQ-OH treated mice many dead worms were recovered and a hepatic shift (i.e. worms migrating to the liver) observed. Hence, FQ and FQ-OH show weak antischistosomal activity in vivo, which is in line with our in vitro results.

\section{Conclusions}

In conclusion, the organometallic compounds evaluated in this study show only weak antischistosomal properties in vivo. Hence, based on our findings an ancillary benefit on schistosomiasis as a result of treating $P$. falciparum infections with FQ is not expected. Furthermore, no correlation can be drawn between the antimalarial and antischistosomal activity of CQ analogues, which might hint to distinct mechanisms of actions. Despite the low activities of organometallic drugs tested against $S$. mansoni so far [25] further derivatives (e.g. organometallic derivatives of MQ) should be studied.

\section{Competing interests}

The authors declare that they have no competing interests.

\section{Authors' contributions}

JK, GG and CB designed the studies. MV and RR carried out the experiments. JK wrote the first draft of the manuscript. GG and CB revised the manuscript. All authors read and approved the final version of the manuscript.

\section{Acknowledgments \\ This work was financially supported by the European Research Council (ERC-2013-CoG 614739-A_HERO to J.K.), the Swiss National Science Foundation (SNSF Professorship PP00P2_133568 to G.G.), the University of Zurich (G.G.), the Stiftung für Wissenschaftliche Forschung of the University of Zurich (G.G.), and the Novartis Jubilee Foundation (R.R. and G.G.). The funders had no role in study design, data collection and analysis, decision to publish, or preparation of the manuscript. Dr. Faustine Dubar is acknowledged for the synthesis of RQ.}

\footnotetext{
Author details

${ }^{1}$ Department of Medical Parasitology and Infection Biology, Swiss Tropical and Public Health Institute, Basel, Switzerland. 'University of Basel, Basel, Switzerland. ${ }^{3}$ Department of Chemistry, University of Zurich, Winterthurerstrasse 190, CH-8057 Zurich, Switzerland. ${ }^{4}$ Université Lille 1, Unité de Glycobiologie Structurale et Fonctionnelle UGSF, F-59650 Villeneuve d'Ascq, France. ${ }^{5}$ CNRS, UMR 8576, F-59650 Villeneuve d'Ascq, France.

Received: 22 August 2014 Accepted: 27 August 2014

Published: 4 September 2014

\section{References}

1. Colley DG, Bustinduy AL, Secor WE, King CH: Human schistosomiasis. Lancet 2014, 383:2253-2264.
}

2. World Health Organisation: Schistosomiasis. In Fact sheet $N^{\circ} 115$. Geneva: World Health Organisation; 2014.

3. Utzinger J, N'Goran EK, Caffrey CR, Keiser J: From innovation to application: Social-ecological context, diagnostics, drugs and integrated control of schistosomiasis. Acta Trop 2011, 120(Suppl 1):S121-S137.

4. Keiser J, Utzinger J: Antimalarials in the treatment of schistosomiasis. Curr Pharm Des 2012, 18:3531-3538.

5. Lee H, Moody-Davis A, Saha U, Suzuki BM, Asarnow D, Chen S, Arkin M, Caffrey CR, Singh R: Quantification and clustering of phenotypic screening data using time-series analysis for chemotherapy of schistosomiasis. BMC Genomics 2012, 13(Suppl 1):S4.

6. Abdulla MH, Ruelas DS, Wolff B, Snedecor J, Lim KC, Xu F, Renslo AR, Williams J, McKerrow JH, Caffrey CR: Drug discovery for schistosomiasis: hit and lead compounds identified in a library of known drugs by medium-throughput phenotypic screening. PLoS Negl Trop Dis 2009, 3:e478.

7. Panic G, Duthaler U, Speich B, Keiser J: Repurposing drugs for the treatment and control of helminth infections. Int J Parasitol Drugs Drug Resist 2014. doi: 10.1016/j.ijpddr.2014.07.002.

8. Keiser J, Chollet J, Xiao SH, Mei JY, Jiao PY, Utzinger J, Tanner M: Mefloquine-an aminoalcohol with promising antischistosomal properties in mice. PloS Negl Trop Dis 2009, 3:e350.

9. Keiser J, N'Guessan NA, Adoubryn KD, Silue KD, Vounatsou P, Hatz C, Utzinger J, N'Goran EK: Efficacy and safety of mefloquine, artesunate, mefloquine-artesunate, and praziquantel against Schistosoma haematobium: randomized, exploratory open-label trial. Clin Infect Dis 2010, 50:1205-1213.

10. Basra A, Mombo-Ngoma G, Melser MC, Diop DA, Wurbel H, Mackanga JR, Furstenau M, Zoleko RM, Adegnika AA, Gonzalez R, Menendez C, Kremsner PG, Ramharter M: Efficacy of mefloquine intermittent preventive treatment in pregnancy against Schistosoma haematobium infection in gabon: a nested randomized controlled assessor-blinded clinical trial. Clin Infect Dis 2013, 56:e68-e75.

11. Biot C, Castro W, Botte CY, Navarro M: The therapeutic potential of metal-based antimalarial agents: implications for the mechanism of action. Dalton Trans 2012, 41:6335-6349.

12. Dubar F, Slomianny C, Khalife J, Dive D, Kalamou H, Guerardel Y, Grellier P, Biot C: The ferroquine antimalarial conundrum: redox activation and reinvasion inhibition. Angew Chem Int Ed Engl 2013, 52:7690-7693.

13. Dubar F, Egan TJ, Pradines B, Kuter D, Ncokazi KK, Forge D, Paul JF, Pierrot C, Kalamou H, Khalife J, Buisine E, Rogier C, Vezin H, Forfar I, Slomianny C, Trivelli X, Kapishnikov S, Leiserowitz L, Dive D, Biot C: The antimalarial ferroquine: role of the metal and intramolecular hydrogen bond in activity and resistance. ACS Chem Biol 2011, 6(3):275-287.

14. Biot C, Daher W, Chavain N, Fandeur T, Khalife J, Dive D, De Clercq E: Design and synthesis of hydroxyferroquine derivatives with antimalarial and antiviral activities. J Med Chem 2006, 49:2845-2849.

15. Keiser J: In vitro and in vivo trematode models for chemotherapeutic studies. Parasitology 2010, 137:589-603.

16. Biot C, Glorian G, Maciejewski LA, Brocard JS: Synthesis and antimalarial activity in vitro and in vivo of a new ferrocene-chloroquine analogue. J Med Chem 1997, 40:3715-3718.

17. Manneck T, Haggenmüller $Y$, Keiser J: Morphological effects and tegumental alterations induced by mefloquine on schistosomula and adult flukes of Schistosoma mansoni. Parasitology 2010, 137:85-98.

18. Xiao SH, Keiser J, Chollet J, Utzinger J, Dong Y, Vennerstrom JL, Tanner M: The in vitro and in vivo activities of synthetic trioxolanes on major human schistosome species. Antimicrob Agents Chemother 2007, 51:1440-1445.

19. AlKadi HO: Antimalarial drug toxicity: a review. Chemotherapy 2007, 53:385-391.

20. Mombo-Ngoma G, Supan C, Dal-Bianco MP, Missinou MA, Matsiegui PB, Ospina Salazar CL, Issifou S, Ter-Minassian D, Ramharter M, Kombila M, Kremsner PG, Lell B: Phase I randomized dose-ascending placebocontrolled trials of ferroquine-a candidate anti-malarial drug-in adults with asymptomatic Plasmodium falciparum infection. Malar J 2011, 10:53.

21. Pomel S, Biot C, Bories C, Loiseau PM: Antiprotozoal activity of ferroquine. Parasitol Res 2013, 112:665-669.

22. Biot C, Nosten F, Fraisse L, Ter-Minassian D, Khalife J, Dive D: The antimalarial ferroquine: from bench to clinic. Parasite 2011, 18:207-214.

23. Xue J, Jiang B, Liu CS, Sun J, Xiao SH: Comparative observation on inhibition of hemozoin formation and their in vitro and in vivo antischistosome activity displayed by 7 antimalarial drugs. Zhongguo $\mathrm{Ji}$ Sheng Chong Xue Yu Ji Sheng Chong Bing Za Zhi 2013, 31:161-169. 
24. Manneck T, Keiser J, Müller J: Mefloquine interferes with glycolysis in schistosomula of Schistosoma mansoni via inhibition of enolase. Parasitology 2012, 139:497-505.

25. Patra M, Ingram K, Leonidova A, Pierroz V, Ferrari S, Robertson MN, Todd $\mathrm{MH}$, Keiser J, Gasser G: In vitro metabolic profile and in vivo antischistosomal activity studies of ( $n(6)$-praziquantel) $\mathrm{Cr}(\mathrm{CO}) 3$ derivatives. J Med Chem 2013, 56:9192-9198.

doi:10.1186/1756-3305-7-424

Cite this article as: Keiser et al:: In vitro and in vivo antischistosomal activity of ferroquine derivatives. Parasites \& Vectors 2014 7:424.

\section{Submit your next manuscript to BioMed Central and take full advantage of:}

- Convenient online submission

- Thorough peer review

- No space constraints or color figure charges

- Immediate publication on acceptance

- Inclusion in PubMed, CAS, Scopus and Google Scholar

- Research which is freely available for redistribution 